\title{
X-ray emission properties of the old pulsar PSR B2224+65
}

\author{
C. Y. Hui and W. Becker
}

\begin{abstract}
Max-Planck Institut für Extraterrestrische Physik, Giessenbachstrasse 1, 85741 Garching bei München, Germany e-mail: dhui@mpe.mpg.de
\end{abstract}

Received 13 October 2006 / Accepted 13 March 2007

\section{ABSTRACT}

\begin{abstract}
Using archival Chandra data we studied the X-ray emission properties of PSR B2224+65 and its environment. Albeit limited by photon statistics the spectral analysis suggests that the bulk of the emission from PSR B2224+65 is non-thermal. Fitting a power-law model to the observed energy spectrum yields a photon index of $\Gamma=1.58_{-0.33}^{+0.43}$. The possible origin of the non-thermal pulsar emission is discussed in the context of the outer-gap model. We did not find any evidence for a compact nebula around PSR B2224+65 though the Chandra data reveal the existence of an extended feature which appears to be associated with PSR B2224+65. It extends from the pulsar position about 2 arcmin to the north-west. Its orientation deviates by $\sim 118^{\circ}$ from the pulsar's proper motion direction. Investigating its energy spectrum shows that the emission of this extended feature is much harder than that of the pulsar itself and is non-thermal in nature.
\end{abstract}

Key words. pulsars: individual: PSR B2224+65 - stars: neutron - X-rays: stars

\section{Introduction}

High energy radiation properties of rotation-powered pulsars are known to approximately correlate with their spin-down age. $\mathrm{X}$-rays from young pulsars with age less than $\sim 10^{5}$ years are believed to be dominated by magnetospheric emission, characterized by a power-law spectrum and narrow pulse profiles. For middle-aged pulsars in the age bracket between $\sim 10^{5}-10^{6}$ years the pulsars' X-radiation is dominated by cooling emission from the hot stellar surface. This class of pulsars is observed to have composite spectra consisting of a soft thermal component, a harder thermal component from heated polar caps as well as some non-thermal emission. The thermal and non-thermal contributions in the emission of younger and middle-aged pulsars has already been well disentangled and studied by previous X-ray observatories (for recent reviews see e.g. Becker \& Aschenbach 2002; Kaspi et al. 2004, and references therein).

Old neutron stars with ages $\gtrsim 10^{6}$ years have already cooled down to less than five hundred thousand degrees so that the thermal emission from their surface has faded from view for X-ray observatories. Recently, the much improved sensitivity of the XMM-Newton and Chandra observatories enabled a series of comprehensive studies to probe and identify the origin of the $\mathrm{X}$-radiation from old rotation-powered pulsars. Until now, results have been presented for the pulsars B0950+08, B0823+26, J2043+2740 (Becker et al. 2004), B0628-28 (Becker et al. 2005), B0943+10 (Zhang et al. 2005), B1133+16 (Kargaltsev et al. 2006) and for PSR B1929+10 (Becker et al. 2006). For all old pulsars which were observed with sufficient photon statistics the X-ray emission turned out to be dominated by nonthermal emission originating in the pulsar's magnetosphere. $\mathrm{X}$-ray pulses, if detected, often show narrow features.

A very interesting member in this class of old pulsars is PSR B2224+65. The proper motion of PSR B2224+65, $\mu_{\mathrm{RA}}=$ $144 \mathrm{mas} / \mathrm{yr}$ and $\mu_{\mathrm{Dec}}=112 \mathrm{mas} / \mathrm{yr}$, is among the highest observed by now (Manchester et al. 2005). The pulsar's interaction with the ISM produces a magnificent bow shock nebula which
Table 1. Proper-motion corrected ephemeris ${ }^{a}$ of PSR B2224+65.

\begin{tabular}{|c|c|}
\hline Right Ascension (J2000) & $22^{\mathrm{h}} 25^{\mathrm{m}} 52.424^{\mathrm{s}}$ \\
\hline Declination (J2000) & $+65^{\circ} 35^{\prime} 34.08^{\prime \prime}$ \\
\hline Pulsar Period, $P(\mathrm{~s})$ & 0.682538228276 \\
\hline Period derivative $\dot{P}\left(10^{-15} \mathrm{~s} \mathrm{~s}^{-1}\right)$ & 9.55 \\
\hline Age $\left(10^{6} \mathrm{yr}\right)$ & 1.13 \\
\hline Surface dipole magnetic field $\left(10^{12} \mathrm{G}\right)$ & 2.58 \\
\hline Epoch of Period (MJD) & 49303.00 \\
\hline $\mathrm{DM}\left(\mathrm{pc} \mathrm{cm}^{-3}\right)$ & 36.079 \\
\hline $\mathrm{DM}_{\text {based distance }}{ }^{(\mathrm{kpc})}$ & 1.86 \\
\hline Spin-down Luminosity $\left(10^{33}\right) \mathrm{erg} \mathrm{s}^{-2}$ & 1.19 \\
\hline
\end{tabular}

${ }^{a}$ Manchester et al. (2005). ${ }^{b}$ According to Cordes \& Lazio (2002).

was discovered in $\mathrm{H} \alpha$ by Cordes et al. (1993). Because the shape of this nebula resembles that of a guitar the nebula got dubbed Guitar Nebula. The pulsar has a period of about $0.68 \mathrm{~s}$ and a period derivative of $9.55 \times 10^{-15} \mathrm{~s} / \mathrm{s}$, implying a spin-down luminosity of $\dot{E}=1.19 \times 10^{33} \mathrm{erg} / \mathrm{s}$ and a spin-down age of somewhat more than one million years. The distance to PSR B2224+65 is not very well constrained. The radio dispersion measure based distance in the Cordes \& Lazio (2002) Galactic free electron density model is $1.86 \mathrm{kpc}$. However, Chatterjee \& Cordes (2004) found by modeling the head of the Guitar Nebula that the pulsar distance could be as close as $1 \mathrm{kpc}$. For this work we adopt the distance of $1 \mathrm{kpc}$ as suggested by Chatterjee \& Cordes (2004). The ephemeris of this pulsar as adopted from Manchester et al. (2005) are summarized in Table 1.

In this paper we present a detailed X-ray study of PSR B2224+65 and its environment based on archival Chandra data. Brief results from this observation were already reported by Wong et al. (2003) and Zavlin \& Pavlov (2004). The structure of this paper is as follows. In Sect. 2 we describe the observations and data analysis and in Sect. 3 we summarize the results 


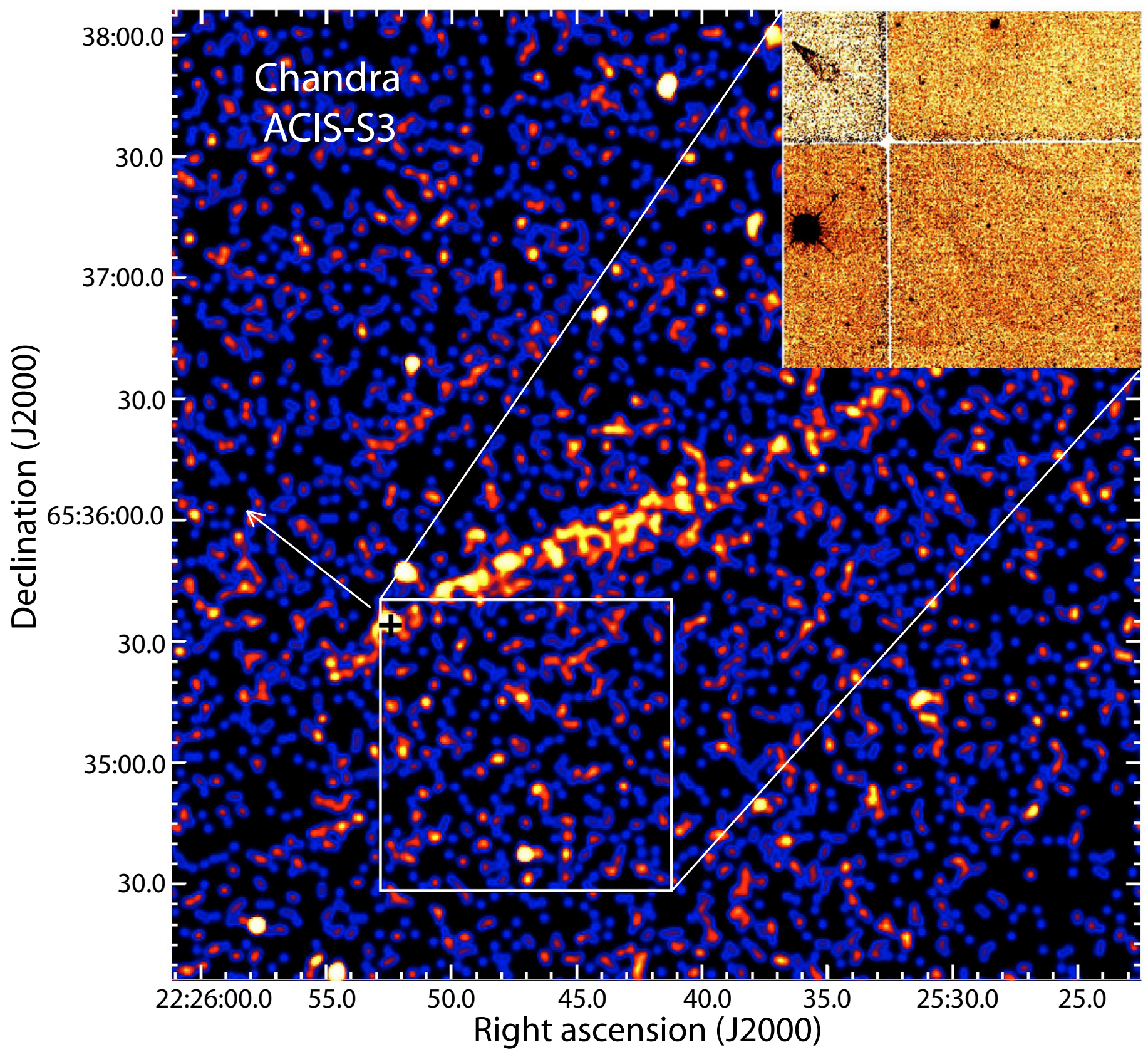

Fig. 1. Chandra ACIS-S3 image in the energy band $0.3-8 \mathrm{keV}$ of the field around PSR B2224+65 smoothed with an adaptive Gaussian filter. The black cross and white arrow indicate the radio pulsar position and the pulsar's proper motion direction. The inset shows the H $\alpha$ image of the Guitar Nebula as seen by HST WFPC2.

which for the pulsar are discussed in the context of the outer-gap model.

\section{Observations and data analysis}

PSR B2224+65 was observed by the Chandra satellite in 2000 October 21-22 (Obs ID: 755) with the Advanced CCD Imaging Spectrometer (ACIS). The pulsar is located on the backilluminated (BI) ACIS-S3 chip which has a superior quantum efficiency among the spectroscopic array. Standard processed level-2 data were used in our study. The effective exposure is found to be $\sim 50 \mathrm{ks}$. The frame time of $3.2 \mathrm{~s}$ does not support a timing analysis of PSR B2224+65 to search for X-ray pulsations.
The Chandra image of the $4 \times 4$ arcmin field around PSR B2224+65 is shown in Fig. 1. The binning factor of the image is 0.5 arcsec. Adaptive smoothing with a Gaussian kernel of $\sigma<2$ pixels has been applied to better make visible faint diffuse emission. The X-ray point source is clearly seen at the radio pulsar position. The inset in Fig. 1 shows the $\mathrm{H} \alpha$ image observed by the Wide Field Planetary Camera 2 (WFPC2) on the Hubble Space Telescope (HST). This image shows the Guitar Nebula. Romani et al. (1997) reported on a $4-\sigma$ detection of diffuse X-ray emission associated with the $\mathrm{H} \alpha$ nebula in ROSAT HRI data. However, we found no evidence for any diffuse X-ray emission within the corresponding region of the Chandra image. Rather than an extended X-ray feature which has a length of $\sim 2$ arcmin but which deviates from the pulsar's direction of proper motion by $\sim 118^{\circ}$ is observed. 


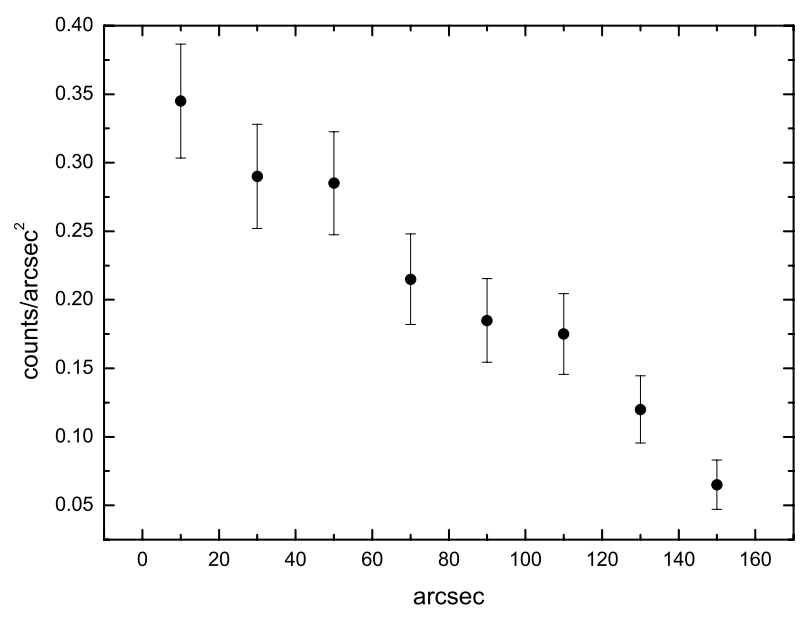

Fig. 2. Vignetting corrected brightness profile in the energy band $0.3-$ $8 \mathrm{keV}$ of the extended feature associated with PSR B2224+65.

The signal-to-noise ratio of this feature in the energy range 2$8 \mathrm{keV}$ is about 6 . We further compute the brightness profile of the feature. From the raw image with bin size of 0.5 arcsec, we estimate counts in eight consecutive boxes of $20 \times 10$ arcsec oriented along the feature. The first box is centered at RA = $22^{\mathrm{h}} 25^{\mathrm{m}} 48.609^{\mathrm{s}}$, Dec $=+65^{\circ} 35^{\prime} 45.15^{\prime \prime}(\mathrm{J} 2000)$. The vignetting corrected brightness profile in the energy band $0.3-8 \mathrm{keV}$ is shown in Fig. 2. The feature was already noticed by Wong et al. (2003). Inspecting the radio data from the NRAO/VLA Sky Survey (NVSS) archive (Condon et al. 1998) did not reveal a radio counterpart of it.

In their brief analysis, Wong et al. (2003) claimed that the $\mathrm{X}$-ray emission at the pulsar position is likely to be from a shocked nebula. In order to investigate whether there is extended nebular emission around PSR B2224+65, we have examined the spatial nature of the emission by fitting a convolved 2-D Gaussian to the sub-image around the pulsar position with the point spread function (PSF) as the convolution kernel. The model PSF at $1.5 \mathrm{keV}$ is created by using the CIAO tool MKPSF. The best-fitted result yields a $F W H M$ of $0.55 \pm 0.03 \operatorname{arcsec}(1-\sigma$ error) which is in agreement with the expected width of the Chandra PSF for a point source observed on-axis.

For a spectral analysis of the pulsar emission we extracted events from a circle of 3 arcsec radius (encircled energy 99\%) centered on the radio pulsar position. The spectrum from the extended X-ray feature was extracted from a box of $132 \times$ 15 arcsec, oriented along the direction of this feature. In total, $\sim 80$ counts for the pulsar and $\sim 370$ counts for the feature were selected in the energy band $0.3-8 \mathrm{keV}$. Even though the photon statistics is on the smaller size it is sufficient to provide us with interesting insights on the radiation emission nature of the pulsar and the unidentified X-ray feature.

Response files were computed by using the CIAO tools MKRMF and MKARF. According to the photon statistics, the spectra were dynamically binned so as to have at least 8 counts per bin for the pulsar and at least 30 counts per bin for the extended feature. The background spectrum for the spectral analysis of the pulsar is extracted from a circle near to the pulsar of 3 arcsec at RA $=22^{\mathrm{h}} 25^{\mathrm{m}} 53.893^{\mathrm{s}}$, Dec $=+65^{\circ} 35^{\prime} 34.79^{\prime \prime}$ (J2000). For the analysis of the extended feature, the background spectrum is extracted from a low count region of $18 \times 15$ arcsec centered at RA $=22^{\mathrm{h}} 25^{\mathrm{m}} 49.113^{\mathrm{s}}$, Dec $=+65^{\circ} 36^{\prime} 08.84^{\prime \prime}(\mathrm{J} 2000)$. In the energy band $0.3-8 \mathrm{keV}$, the net count rates for the pulsar and the feature are estimated to be $(1.53 \pm 0.18) \times 10^{-3} \mathrm{cts} / \mathrm{s}$
Table 2. Spectral parameters inferred from fitting the Chandra observed spectrum of PSR B2224+65 and X-ray jet.

\begin{tabular}{|c|c|c|c|c|c|}
\hline Model $^{a}$ & $\chi_{v}^{2}$ & d.o.f. & $\begin{array}{c}N_{\mathrm{H}} \\
10^{21} \mathrm{~cm}^{-2}\end{array}$ & $\Gamma / k T$ & Normalization $^{b}$ \\
\hline \multicolumn{6}{|c|}{ PSR B2224+65 } \\
\hline PL & 0.70 & 6 & $\leq 0.90$ & $1.58_{-0.33}^{+0.43}$ & $2.28_{-0.39}^{+0.75} \times 10^{-6}$ \\
\hline BB & 2.07 & 6 & 0.00 & $0.46^{-0.33}$ & $1.46 \times 10^{-2}$ \\
\hline \multicolumn{6}{|c|}{ Extended X-ray feature } \\
\hline PL & 0.79 & 9 & $\leq 2.25$ & $0.90_{-0.24}^{+0.35}$ & $5.66_{-1.31}^{+3.56} \times 10^{-6}$ \\
\hline BB & 1.50 & 9 & $\leq 0.62$ & $0.91_{-0.14}^{+0.15}$ & $7.17_{-2.68}^{+4.82} \times 10^{-3}$ \\
\hline BREMSS & 0.86 & 9 & $1.47_{-1.02}^{+1.44}$ & $199.36_{-199.36}^{+0.64}$ & $2.46_{-0.99}^{+0.38} \times 10^{-5}$ \\
\hline
\end{tabular}

$a \mathrm{PL}=$ power-law; $\mathrm{BB}=$ blackbody; BREMSS $=$ Thermal bremsstrahlung ${ }^{b}$ The entry in this column depends on the model in interest. For the power-law model, the unit of the normalization constant is photons $\mathrm{keV}^{-1} \mathrm{~cm}^{-2} \mathrm{~s}^{-1}$. For the blackbody model, the normalization is $\left(R_{\mathrm{km}} / D_{10}\right)^{2}$, where $R_{\mathrm{km}}$ is the source radius in $\mathrm{km}$ and $D_{10}$ is the distance to the source in unit of $10 \mathrm{kpc}$. For the thermal bremsstrahlung model, the normalization constant is expressed as $\left(3.02 \times 10^{-15} / 4 \pi D^{2}\right) \int n_{\mathrm{e}} n_{\mathrm{I}} \mathrm{d} V$ where $D$ is the source distance in $\mathrm{cm}$ and $n_{\mathrm{e}}$ and $n_{\mathrm{I}}$ are the electron and ion densities in $\mathrm{cm}^{-3}$.

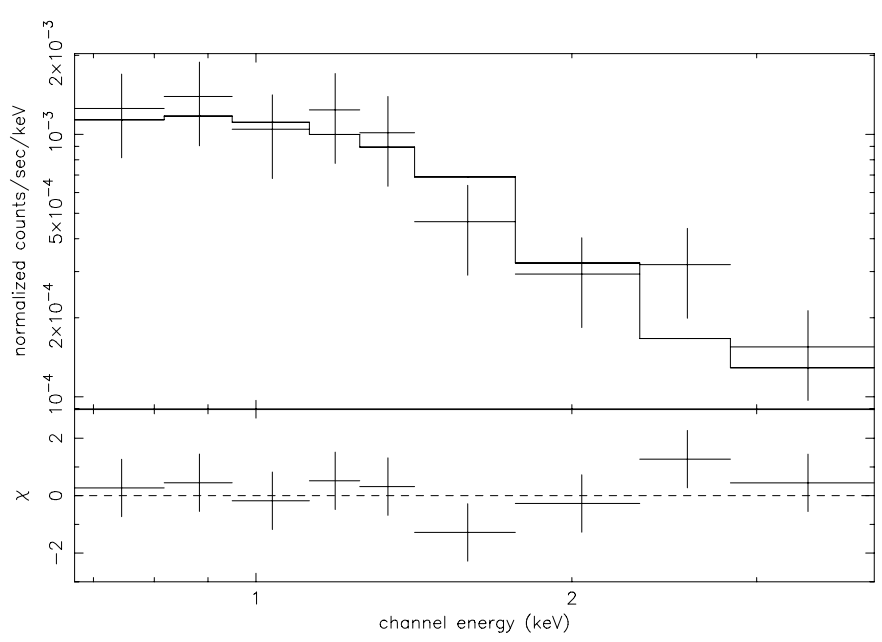

Fig. 3. Energy spectrum of PSR B $2224+65$ as observed with the Chandra ACIS-S3 detector and fitted to an absorbed power-law model (upper panel) and contribution to the $\chi^{2}$ fit statistic (lower panel).

and $(5.59 \pm 0.34) \times 10^{-3} \mathrm{cts} / \mathrm{s}$ respectively. With the aid of PIMMS, the pileup fraction of the ACIS-S3 data is estimated to be $\lesssim 0.7 \%$ which is negligible. All spectral fits were performed in the $0.3-8 \mathrm{keV}$ energy range by using XSPEC 11.3.1. The degradation of the ACIS quantum efficiency was corrected by the XSPEC model ACISABS. The parameters of all fitted model spectra are summarized in Table 2 . All the quoted errors are $1-\sigma$ and were computed for 1 parameter in interest.

For the pulsar PSR B2224+65 we found that among the tested models a single power-law model describes the observed spectrum best $\left(\chi_{v}^{2}=0.70\right.$ for 6 d.o.f.). This model yields a column density of $N_{\mathrm{H}}<0.9 \times 10^{21} \mathrm{~cm}^{-2}$, a photon index of $\Gamma=1.58_{-0.33}^{+0.43}$ and a normalization at $1 \mathrm{keV}$ of $\left(2.28_{-0.39}^{+0.75}\right) \times$ $10^{-6}$ photons $\mathrm{keV}^{-1} \mathrm{~cm}^{-2} \mathrm{~s}^{-1}$. The best-fitted power-law spectrum and residuals are shown in Fig. 3.

The unabsorbed fluxes deduced for the best-fitting power-

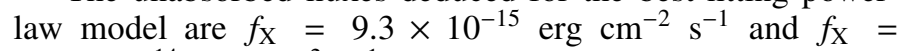
$1.6 \times 10^{-14} \mathrm{erg} \mathrm{cm}^{-2} \mathrm{~s}^{-1}$ in the $0.1-2.4 \mathrm{keV}$ and $0.510 \mathrm{keV}$ energy bands, respectively. The luminosities in these bands are 


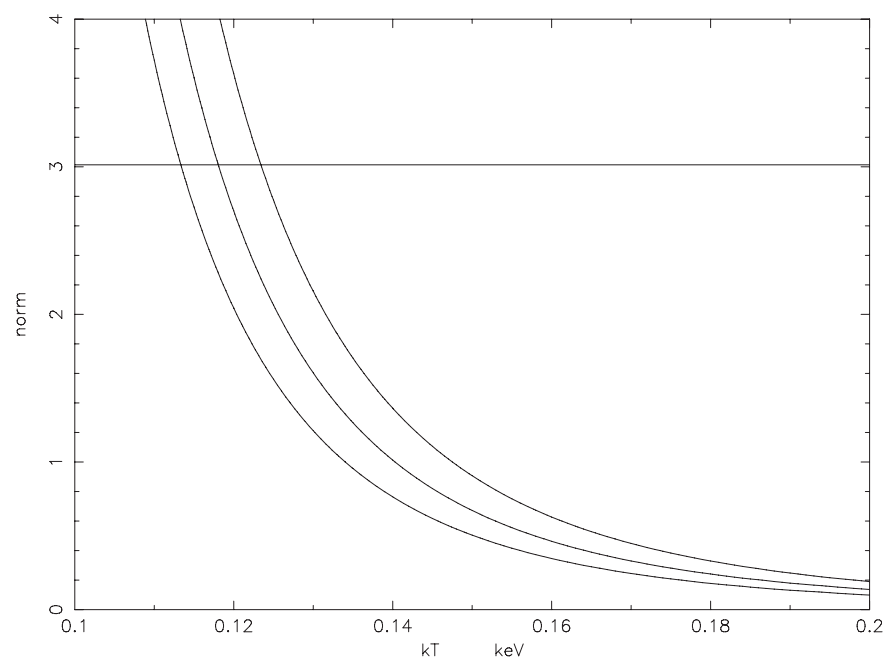

Fig. 4. Portion of the $1 \sigma, 2 \sigma$ and $3 \sigma$ confidence contours showing the blackbody normalization versus temperature for a blackbody model added on the best-fitted power law model.The horizontal line at a normalization of 3.06 corresponds to a polar cap radius of $175 \mathrm{~m}$ and a pulsar distance of $1 \mathrm{kpc}$ as suggested by Chatterjee \& Cordes (2004).

$L_{\mathrm{X}}=1.1 d_{1 \mathrm{kpc}}^{2} \times 10^{30} \mathrm{erg} \mathrm{s}^{-1}$ and $L_{\mathrm{X}}=2.0 d_{1 \mathrm{kpc}}^{2} \times 10^{30} \mathrm{erg} \mathrm{s}^{-1}$. $d_{1 \mathrm{kpc}}$ denotes the distance to PSR B2224+65 in units of $1 \mathrm{kpc}$. The conversion efficency, $L_{\mathrm{X}}(0.1-2.4 \mathrm{keV}) / \dot{E}$, is found to be $0.9 d_{1 \mathrm{kpc}}^{2} \times 10^{-3}$. For a distance close to $1 \mathrm{kpc}$ as suggested by Chatterjee \& Cordes (2004), this is in good agreement with the relation $L_{\mathrm{X}}(0.1-2.4 \mathrm{keV}) \approx 10^{-3} \dot{E}$ found by Becker \& Trümper (1997) for rotation powered pulsars.

We have also considered the possibility of a purely thermal emission scenario. However, fitting a blackbody yields a reduced $\chi_{v}^{2}$ of 2.06 for 6 d.o.f. which invalidates this model. Although the small number of counts does not support any fitting with multicomponent models, we were still able to estimate the upper limit for the polar cap temperature and surface temperature by adding a blackbody component to the best-fitting powerlaw model. We computed the confidence contours of the blackbody normalization versus the temperature. They are shown in Fig. 4. Assuming a dipolar magnetic field, the conventional size of a polar cap is defined by the last open field lines. The radius of the polar cap area is given as $r_{\mathrm{pc}}=R(2 \pi R / c P)^{1 / 2}$, where $R$ is the neutron star radius, $c$ is the speed of light and $P$ is the rotation period of the pulsar. For PSR B2224+65, the rotation period of $0.68 \mathrm{~s}$ implies a polar cap of radius $r_{\mathrm{pc}}=175 \mathrm{~m}$. With this estimate we set a $1-\sigma$ polar cap temperature upper limit of $T_{\mathrm{pc}}^{\infty}<1.3 \times 10^{6} \mathrm{~K}$ by assuming contribution from one polar cap only. This corresponds to a $1-\sigma$ upper limit on the bolometric luminosity of $L_{\mathrm{bol}}^{\mathrm{pc}}<6.5 \times 10^{29} \mathrm{erg} \mathrm{s}^{-1}$. Zavlin \& Pavlov (2004) have estimated a $1-\sigma$ upper limit on the bolometric luminosity of $L_{\mathrm{bol}}^{\mathrm{pc}}<9.1 \times 10^{29} \mathrm{erg} \mathrm{s}^{-1}$ by adopting a hydrogen polar cap model. However, such models have many free parameters of which most are unknown for PSR B2224+65 (e.g. magnetic inclination, viewing angle). In view of this, the applicability of this model is restricted and a simple blackbody model regarded to provide us with a more conservative upper limit. If we assume that the thermal emission is emitted from the whole neutron star surface of $10 \mathrm{~km}$ radius we compute a surface temperature upper limit to be $T_{\mathrm{s}}^{\infty}<6.1 \times 10^{5} \mathrm{~K}(3-\sigma)$. This limit is of the same order as those found for the other old X-ray detected pulsars (see Becker et al. 2004, 2005, 2006).

For the linearly extended feature we found that the spectrum is best-fitted by a single power-law model $\left(\chi_{v}^{2}=0.79\right.$

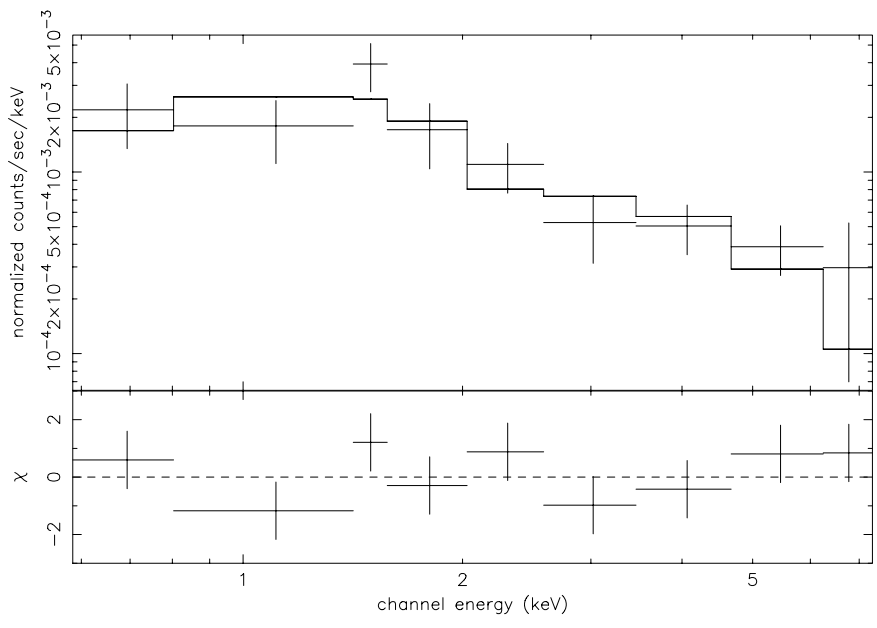

Fig. 5. Energy spectrum of extended feature detected in the field of PSR B2224+65, as observed with Chandra ACIS-S3 detector and fitted to an absorbed power-law model (upper panel) and contribution to the $\chi^{2}$ fit statistic (lower panel).

for 9 d.o.f.). This suggests a non-thermal origin of its emission. The model inferred column absorption is found to be $N_{\mathrm{H}}<$ $2.25 \times 10^{21} \mathrm{~cm}^{-2}$. The photon index is $\Gamma=0.90_{-0.24}^{+0.35}$ and the normalization at $1 \mathrm{keV}$ is $\left(5.66_{-1.31}^{+3.56}\right) \times 10^{-6}$ photons $\mathrm{keV}^{-1} \mathrm{~cm}^{-2} \mathrm{~s}^{-1}$. The best-fitted power-law spectrum and residuals are shown in Fig. 5. The unabsorbed fluxes of the extended feature deduced for the best-fitted model parameters and the energy ranges $0.1-2.4 \mathrm{keV}$ and $0.5-10 \mathrm{keV}$ are $f_{\mathrm{X}}=2.1 \times 10^{-14} \mathrm{erg} \mathrm{cm}^{-2} \mathrm{~s}^{-1}$ and $f_{\mathrm{X}}=1.0 \times 10^{-13} \mathrm{erg} \mathrm{cm}^{-2} \mathrm{~s}^{-1}$, respectively. The corresponding luminosities in the $0.1-2.4 \mathrm{keV}$ and $0.5-10 \mathrm{keV}$ bands are $L_{\mathrm{X}}=2.5 d_{1 \mathrm{kpc}}^{2} \times 10^{30} \mathrm{erg} \mathrm{s}^{-1}$ and $L_{\mathrm{X}}=1.2 d_{1 \mathrm{kpc}}^{2} \times 10^{31} \mathrm{erg} \mathrm{s}^{-1}$, respectively. Fitting the spectrum of the X-ray feature with the blackbody model does not yield an acceptable goodness-of-fit $\left(\chi_{v}^{2}=1.50\right.$ for 9 d.o.f.). We have also tried to fit the spectrum with a thermal bremsstrahlung model. However, the model implies a temperature as high as $200 \mathrm{keV}$ and hence can be rejected simply because it is not physical.

\section{Discussion and conclusion}

According to our analysis, the observed X-rays of PSR B2224+65 are of non-thermal origin, suggesting a magnetospheric radiation dominated emission scenario. To model the detected X-ray spectrum with a blackbody model yields no acceptable results. A distinct extended X-ray feature of 2 arcmin size is observed to start at the pulsar position. Its $\mathrm{X}$-ray spectrum is very hard and no counterpart of it is found in $\mathrm{H} \alpha$ nor in NVSS radio data. Comparing the energy spectra of PSR B2224+65 and of the extended feature it is obvious that the X-ray emission of the later is much harder. We found that neither a blackbody nor a thermal bremsstrahlung model yields a valid description for its observed spectrum. This contradicts to the findings reported by Wong et al. (2003) who fitted the emission from the extended feature with a blackbody. That the $\mathrm{X}$-ray feature is not aligned with the pulsar's proper motion does not a priori invalidate its association with the pulsar. Markwardt \& Ögelman (1995) reported the ROSAT PSPC observation of a highly extended jet-like feature associated with the Vela pulsar which also is found to be entirely misaligned with the pulsar's proper motion vector. The recent discovery of the X-ray trail associated with the millisecond pulsar PSR J2124-3358 is another example (Hui \& Becker 2006). Such asymmetry might 


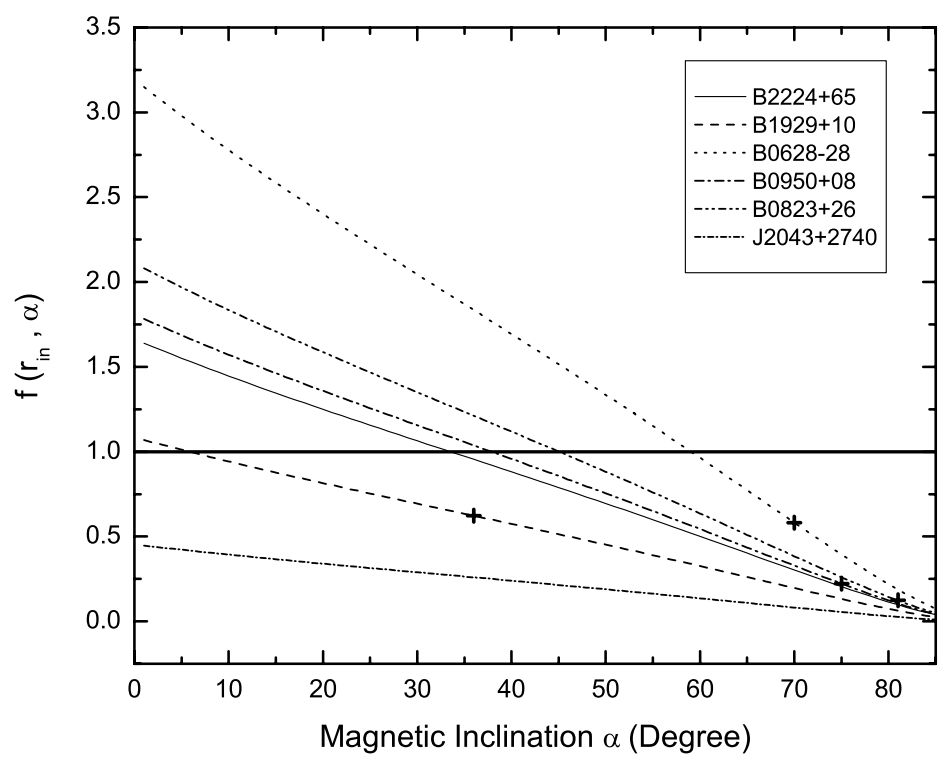

Fig. 6. Variation of the fractional size of the outer-gap at the null charge surface with magnetic inclination angle for old pulsars. The crosses indicate the calculated $f\left(r_{\mathrm{in}}, \alpha_{\mathrm{RVM}}\right)$ for the pulsars with measured magnetic inclination angle (cf. Table 3$)$.

Table 3. The calculated $f_{0}$ and $f\left(r_{\mathrm{in}}, \alpha_{\mathrm{RVM}}\right)$ of old pulsars according to CZ99 and Zhang et al. (2004) respectively.

\begin{tabular}{lccccc}
\hline \hline PSR & $P(\mathrm{~s})$ & $B\left(10^{12} \mathrm{G}\right)$ & $\alpha_{\mathrm{RVM}}(\text { degree })^{a}$ & $f_{0}=5.5 P^{26 / 21} B_{12}^{-4 / 7}$ & $f\left(r_{\text {in }}, \alpha_{\mathrm{RVM}}\right)$ \\
\hline B2224+65 & 0.68 & 2.58 & - & 1.99 & - \\
B1929+10 & 0.23 & 0.51 & 35.97 & 1.31 & 0.62 \\
B0628-28 & 1.24 & 3.02 & 70 & 3.82 & 0.58 \\
B0950+08 & 0.25 & 0.25 & 74.6 & 2.18 & 0.23 \\
B0823+26 & 0.53 & 0.98 & 81.1 & 2.54 & 0.12 \\
J2043+2740 & 0.096 & 0.35 & - & 0.55 & - \\
\hline
\end{tabular}

${ }^{a}$ The magnetic inclination angles $\alpha_{\mathrm{RVM}}$ are deduced from fitting the rotating vector model (RVM) to the radio polarization angle swing. $\alpha_{\mathrm{RVM}}$ of B0628-28 is taken from Becker et al. (2005). Others are adopted from Everett \& Weisberg (2001).

be caused by e.g. the inhomogeneities of the surrounding ISM or anisotropies in the pulsar wind and/or bulk flow of ambient gas (see also Gaensler, Jones \& Stappers 2002). The fact that the feature starts exactly at the position of PSR B2224+65 argues against an interpretation as random ISM feature. Deeper future observations though are badly needed to further study this unique system.

Same as PSR B2224+65 the X-ray emission of many old pulsars are found to be described by power-law spectral models (Becker et al. 2004, 2005, 2006) suggesting that their X-ray emission is largely dominated by non-thermal emission processes. This is further supported by the fact that the pulse profiles of this class of pulsars are not sinusoidal as it would be expected from spin-modulated thermal emission (cf. Becker et al. 2004, 2005, 2006). For B1133+16 (Kargaltsev et al. 2006) and B0943+10 (Zhang et al. 2005), Owing to the limited photon statistics, their spectra can be fitted equally well by both blackbody and power-law models. While we cannot discriminate whether the X-ray emission are thermal or non-thermal in these cases, we have not taken these two pulsars in the following discussion.

According to Cheng \& Zhang (1999; hereafter CZ99), the non-thermal X-rays from pulsars are synchrotron radiation of electron-positron pairs which are created in the strong magnetic field near the neutron star surface by curvature photons. These photons are emitted by charged particles on their way from the outer-gap to the stellar surface. The fractional size of the outergap, $f_{0}$, is defined as the ratio between the mean vertical separation of the gap boundaries in the plane spanned by the rotation axis and the magnetic axis to the light cylinder radius. CZ99 estimated the fractional gap size as $f_{0}=5.5 P^{26 / 21} B_{12}^{-4 / 7}$ and determined the existence of the outer-gap by the criteria $f_{0}<1$. We calculated the $f_{0}$ for six recently studied old pulsars and tabulated them in Table 3 . Five out of six pulsars have $f_{0}>1$ which indicates that all but one of these old pulsars do not have outergaps in the framework of this model. Rather then, the CZ99model predicts that if there is any X-ray emission from these old pulsars, it should be thermal. This is obviously not in agreement with the observations, showing the limitation of this model.

A point, however, which has not been considered in this model is that the fractional gap size should depend on the inclination angle $\alpha$ of the magnetic axis vs. the rotational axis. As mentioned by Zhang et al. (2004), the active region of the outergap should begin at the null charge surface $(\Omega \cdot B=0)$ which is at the radial distance $r_{\text {in }}$ from the star. Since the fractional size reaches a minimum at $r_{\text {in }}$, it is more reasonable to determine whether the gap exist by the criteria $f\left(r_{\text {in }}, \alpha\right)<1$ rather than by $f_{0}<1$. Especially for larger magnetic inclination angles the null charge surface is expected to move closer to the star, i.e. $f\left(r_{\text {in }}, \alpha\right)$ decreases with increasing $\alpha$. Using Eq. (36) in Zhang et al. (2004) we calculated the variation of $f\left(r_{\text {in }}, \alpha\right)$ with $\alpha$ for several X-ray detected old pulsars and plotted the result in Fig. 6. Interestingly, for PSRs B0950+08, B0823+26, B062828 and B1929+10, the inclination angles deduced from fitting the rotating vector model to the radio polarization angle swing (Everett \& Weisberg 2001; Becker et al. 2005) are all in the region $f\left(r_{\text {in }}, \alpha\right)<1$ (c.f. Fig. 6 and Table 3 ). The emission geometry for the PSR B2224+65 is currently unknown which prevents us to discuss whether the outer-gap can sustain in this pulsar. 
Whether the outer-gap model is of any general meaning for the description of the X-ray emission from old pulsars can only be constrained by observations. It is necessary to obtain the light curves and the spectra from a much larger sample than currently available. By now it seems rather speculative to assume that those X-ray detected old pulsars, by chance, all have their inclination angles in that range for which the outer-gap model predicts non-thermal emission.

Acknowledgements. We thank the referee David Helfand for thoroughly reading the manuscript and the many useful comments.

\section{References}

Becker, W., \& Trümper, J. 1997, A\&A, 326, 682

Becker, W., \& Aschenbach, B. 2002, in Proceedings of the WE-Heraeus Seminar on Neutron Stars, Pulsars and Supernova remnants, ed. W. Becker, H. Lesch, \& J. Trümper, MPE-Rep. 278, 64, (available from [arXiv:astro-ph/0208466])

Becker, W., Weisskopf, M. C., Tennant, et al. 2004, ApJ, 615, 908

Becker, W., Jessner, A., Kramer, M., Testa, V., \& Howaldt, C. 2005, ApJ, 633,
Becker, W., Kramer, M., Jessner, A., et al. 2006, ApJ, 645, 1421

Chatterjee, S., \& Cordes, J. M. 2004, ApJ, 600, L51

Cheng, K. S., \& Zhang, L. 1999, ApJ, 515, 337

Condon, J. J., Cotton, W. D., Greisen, E. W., et al. 1998, AJ, 115, 1693

Cordes, J. M., \& Lazio, T. J. W., 2002 [arXiv: astro-ph/0207156]

Cordes, J. M., Romani, R. W., \& Lundgren, S. C. 1993, Nature, 362, 133

Everett, J. E., \& Weisberg, J. M. 2001, ApJ, 553, 341

Gaensler, B. W., Jones, D. H., \& Stappers, B. W. 2002, ApJ, 580, L137

Hui, C. Y., \& Becker, W. 2006, A\&A, 448, L13

Kargaltsev, O., Pavlov, G. G., \& Garmire, G. P. 2006, ApJ, 636, 406

Kaspi, V. M., Roberts, M. S. E., \& Harding, A. 2004 [arXiv: astro-ph/0402136]

Manchester, R. N., Hobbs, G. B., Teoh, A., \& Hobbs, M. 2005, AJ, 129, 1993

Markwardt, C. B., \& Ögelman, H. 1995, Nature, 375, 40

Romani, R. W., Cordes, J. M., \& Yadigaroglu, I.-A. 1997, ApJ, 484, L137

Wong, D. S., Cordes, J. M., Chatterjee, S., et al. 2003, High Energy Processs and Phenomena in Astrophysics, ed. X. D. Li, V. Trimble, \& Z. R. Wang, IAU Symp., 214, 135

Zavlin, V. E., \& Pavlov, G. G. 2004, ApJ, 616, 452

Zhang, B., Sanwal, D., \& Pavlov, G. G. 2005, 624, L109

Zhang, L., Cheng, K. S., Jiang, Z. J., \& Leung, P. 2004, ApJ, 604, 317 367 